\title{
A UNIQUENESS RESULT FOR TOPOLOGICAL GROUPS
}

\author{
ROBERT R. KALLMAN 1
}

\begin{abstract}
We give a rapid proof of a general result which has an easy corollary that the $p$-adic integers have a unique topology in which they are a complete separable metric group.
\end{abstract}

In [1] Corwin showed that the p-adic integers have a unique topology in which they are a nondiscrete locally compact group. The purpose of this note is to give a rapid proof of the following general theorem. It contains most of Corwin's result as a special case. The proof is by methods different than those employed by Corwin.

TheOREM 1. Let $G$ be a complete separable Abelian metric group. For each integer $n$, let $n \cdot G=[n a \mid a$ in $G]$. Suppose that the translates of the $n \cdot G$ generate the Borel structure of $G$. Then $G$ has a unique topology in which it is a complete separable metric group.

Proof. It is not a priori obvious that the $n \cdot G$ are Borel subsets of $G$. However, let $L$ and $K$ be complete separable metric groups, and $\psi: L \rightarrow K$ a continuous homomorphism. Then $\psi$ induces a continuous one-to-one homomorphism of $L /$ kernel $\psi$ onto $\psi(L)$. Since $L /$ kernel $\psi$ is also a complete separable metric group, Souslin's theorem implies that $\psi(L)$ is a Borel subset of $K$. In particular, the $n \cdot G$ are Borel subsets of $G$.

Let $G^{\prime}$ be a complete separable metric group which is isomorphic to $G$ as an abstract group but perhaps has a different topology. Let $\phi: G^{\prime} \rightarrow G$ be the natural identification. But for each integer $n, n \cdot G^{\prime}=\phi^{-1}(n \cdot G)$ is a Borel subset of $G^{\prime}$. Hence, since the translates of the $n \cdot G$ generate the Borel structure of $G$, we have that $\phi$ is a Borel mapping. Hence, by Kuratowski [2, p. 400], there exists a set $P$ of first category in $G^{\prime}$ such that $\phi \mid G^{\prime}-P$ is continuous.

The proof of the theorem may now be completed in standard fashion. We claim that $\phi$ is actually continuous on all of $G^{\prime}$. To show this, let $a_{n}(n \geq 1)$ and $a$ be elements of $G^{\prime}$ such that $a_{n} \rightarrow a($ as $n \uparrow \infty)$. Now if $Q$ is the set which is the union of $a^{-1} \cdot P$ and $a_{n}^{-1} \cdot P(n \geq 1), Q$ is again a set of the first category. Hence, $G^{\prime}-Q$ is nonempty. Let $b$ be an element of $G^{\prime}-Q$. Then $a b$ is in $G^{\prime}-P$ and $a_{n} b$ is in $G^{\prime}-P(n \geq 1)$. But $a_{n} b \rightarrow a b$. Hence, $\phi\left(a_{n} b\right) \rightarrow \phi(a b)$, and so $\phi\left(a_{n}\right)=\phi\left(a_{n} b\right) \cdot \phi\left(b^{-1}\right) \rightarrow \phi(a b) \cdot \phi\left(b^{-1}\right)=\phi(a)$. Hence, $\phi$ is a continuous one-to-one mapping of $G^{\prime}$ onto $G$. Hence, since both

Received by the editors May 14, 1975.

AMS (MOS) subject classifications (1970). Primary 22D05; Secondary $22 \mathrm{E} 35$.

Key words and phrases. Topological groups, $p$-adic integers.

${ }^{1}$ Supported in part by NSF Grant GP-38023. 
$G$ and $G^{\prime}$ are complete separable metric groups, $\phi$ is actually a topological isomorphism. Q.E.D.

Note that Corwin's result is an immediate corollary. If $G$ is the $p$-adic integers, then the $n \cdot G$ are open subgroups of $G$ which form a basis at the identity. Hence, the translates of the $n \cdot G$ generate the topology and thus the Borel structure of $G$.

\section{BIBLIOGRAPHY}

1. L. Corwin, Uniqueness of topology for the p-adic integers, Proc. Amer. Math. Soc. (to appear).

2. K. Kuratowski, Topology. Vol. 1, 5th ed., PWN, Warsaw; Academic Press, New York, 1966. MR 36 \#840.

Department of Mathematics, University of Florida, Gainesville, Florida 32611 